\title{
STRESS MEASURES IN SOM LEARNING
}

\author{
Zuzana Krbcova $^{1 *}$, Jaromir Kukal ${ }^{1}$ \\ ${ }^{1}$ University of Chemistry and Technology in Prague \\ Faculty of Chemical Engineering \\ Department of Computing and Control Engineering \\ Technicka 5, 16626 Prague 6 \\ Czech Republic
}

\begin{abstract}
Various stress measures can be used in generalized version of Sammon's mapping. Kohonen SOM with iterative or batch learning is a standard tool for data self-organization, too. Our method applies stress functions to pattern relationships in SOM and converts batch learning to discrete optimization task. Due to NP-completeness of SOM learning, optimization heuristics have to be used. Simulated annealing making use of Lévy flights is the recommended heuristics for this task.
\end{abstract}

Keywords: SOM, metric space, stress function, optimization heuristics

\section{Introduction}

Objects represented by matrix of mutual distances are mapped into a structure of self organizing map so as to minimize stress functions. Optimization heuristic is applied to the finding minimum of stress measures. The following notation is used in this paper: let $\mathbb{A}, \mathbb{B}$ be non-empty finite sets representing input and output data spaces. In many applications $\mathbb{A}=\mathbb{R}^{M}, \mathbb{B}=\mathbb{R}^{m}$, where $M>m$ and Euclidean metrics is used. However our aim is to design a novel method using SOM [1, 2] topology. The output structure of SOM [3, 4] can be represented by graph $\mathcal{G}$, where $\mathcal{G}=\langle\mathbb{V}, \mathbb{E}\rangle$ is undirected connected graph consisting of vertex set $\mathbb{V}=\mathrm{V}(\mathcal{G})$, edge set $\mathbb{E}=\mathrm{E}(\mathcal{G}) \subset\left(\begin{array}{c}\mathbb{V} \\ 2\end{array}\right)$, and cardinality of $\mathrm{V}(\mathcal{G})$ is $H$. The distance between two vertices $k, l \in \mathbb{V}$ is measured by natural metric $d_{\mathrm{G}}(k, l)$, which is defined as the length of shortest path connecting vertices $k$ and $l$. Therefore, in our case $\mathbb{B}=\mathrm{V}(\mathcal{G})$. Let $\langle\mathbb{A}, \delta\rangle,\left\langle\mathrm{V}(\mathcal{G}), d_{\mathrm{G}}\right\rangle$ be two metric spaces. Let $C$ be the number of patterns in $\mathbb{A}$. Square matrix $\mathbf{D}=\left(D_{i j}\right)_{i, j=1}^{C}$ represents the distances between each pair of elements $\mathbf{a}_{i}, \mathbf{a}_{j} \in \mathbb{A}$, thus $D_{i j}=\delta\left(\mathbf{a}_{i}, \mathbf{a}_{j}\right)$. Similarly, square matrix $\mathbf{L}=\left(L_{i j}\right)_{i, j=1}^{C}$ represents the distances between the corresponding mapped elements of set $\mathrm{V}(\mathcal{G})$ as $L_{i j}=d_{\mathrm{G}}\left(\mathbf{b}_{p_{i}}, \mathbf{b}_{p_{j}}\right)$, where vector $\mathbf{p} \in\{1, \cdots, H\}^{C}$ is a vector assigning $i$-th element of $\mathbb{A}$ to $p_{i}$-th element of $\mathbb{B}$ and will be the subject of optimization.

\section{Portfolio of Stress Functions}

Metric multidimensional scaling (MMDS) includes a stress function which can be generalized by function $\mathrm{E}_{\mathrm{MMDS}}$. The form of function $\mathrm{E}_{\mathrm{MMDS}}$ is

$$
E_{\mathrm{MMDS}}=\frac{1}{S} \sum_{i<j}\left(L_{i j}-D_{i j}\right)^{2} w_{i j},
$$

where $S>0$ is normalization scalar and $w_{i j} \geq 0$ is weight of $D_{i j}$ in general. We use particular stress functions of MMDS [5]:

- Raw-stress: $E_{\mathrm{RS}}=\sum_{i<j}\left(L_{i j}-D_{i j}\right)^{2}$

- Sammon: $E_{\mathrm{SA}}=\frac{1}{\sum_{i<j} D_{i j}} \sum_{i<j} \frac{\left(L_{i j}-D_{i j}\right)^{2}}{D_{i j}}$

and several stress functions of Bregmanised MMDS [6, 7]:

- Itakura-Saito: $E_{\mathrm{IS}}=\sum_{i<j}\left(\frac{L_{i j}}{D_{i j}}-\ln \frac{L_{i j}}{D_{i j}}-1\right)$

- Reciprocal: $E_{\mathrm{RE}}=\sum_{i<j}\left(\frac{1}{L_{i j}}-\frac{2}{D_{i j}}+\frac{L_{i j}}{D_{i j}^{2}}\right)$

- Inverse quadratic: $E_{\mathrm{IQ}}=\sum_{i<j}\left(\frac{1}{L_{i j}^{2}}-\frac{3}{D_{i j}^{2}}+\frac{2 L_{i j}}{D_{i j}^{3}}\right)$

- Extended Sammon: $E_{\mathrm{ESA}}=\sum_{i<j}\left(L_{i j} \ln \frac{L_{i j}}{D_{i j}}-L_{i j}+D_{i j}\right)$. 


\section{Self Organizing Mapping and Its Stress}

An input metric space is frequently linear with Euclidean distance. Comparison of the Euclidean distance $D_{i j}$ with natural graph distance $L_{i j}=d_{G}\left(\mathbf{b}_{p_{i}}, \mathbf{b}_{p_{j}}\right)$ enforced graph distance scaling by formula $L_{i j}^{\text {new }}=\alpha L_{i j}$, where $\alpha>0$ is defined as minimizer of each stress function using explicit formulas

- $E_{\mathrm{RS}}: \alpha=\frac{\sum_{i<j} L_{i j} D_{i j}}{\sum_{i<j} L_{i j}^{2}}$

- $E_{\mathrm{RE}}: \alpha=\sqrt{\frac{\sum_{i<j} L_{i}}{\sum_{i<j} \frac{L_{i j}}{D_{i j}}}}$

- $E_{\mathrm{SA}}: \alpha=\frac{\sum_{i<j} L_{i j}}{\sum_{i<j} \frac{L_{i j}^{2}}{D_{i j}}}$

- $E_{\mathrm{IQ}}: \alpha=\left(\frac{\sum_{i<j} L_{i j}^{-2}}{\sum_{i<j} \frac{L_{i j}}{D_{i j}^{3}}}\right)^{1 / 3}$

- $E_{\mathrm{IS}}: \alpha=\frac{(C-1) C}{2 \sum_{i<j} \frac{L_{i j}}{D_{i j}}}$

- $E_{\mathrm{ESA}}: \alpha=\exp \left(-\frac{\sum_{i<j} L_{i j} \ln \frac{L_{i j}}{D_{i j}}}{\sum_{i<j} L_{i j}}\right)$.

This data scaling does not change distance proportions. The resulting stress functions are then optimized with respect to real parameter $\alpha$. Therefore mixed integer optimization tasks are converted to discrete optimization ones with unknown partition $\mathbf{p}$.

\section{Partition Optimization}

Heuristic optimization technique with embedded Lévy flights [8] was used for integer minimization of stress and correlation measures. The technique is similar to Fast Simulated Annealing (FSA) [9] but Cauchy distribution is used in mutation operator instead of an acceptance decision. We also used fixed temperature according to [8].

Supposing optimization domain is $\mathbb{D}=\{1, \cdots, H\}^{C}$, we minimize the objective function $\mathrm{f}: \mathbb{D} \rightarrow \mathbb{R}_{0}^{+}$. The optimum value is denoted as $\mathrm{f}^{*}=\min _{\mathbf{p} \in \mathbb{D}} \mathrm{f}(\mathbf{p})$, and, therefore, we find any point satisfying

$$
\mathbf{p}_{\text {opt }} \in \underset{\mathbf{p} \in \mathbb{D}}{\arg \min } \mathrm{f}(\mathbf{p})
$$

Integer heuristics description uses three basic terms: uniform distribution on any point set is denoted as $\mathrm{U}(\cdots)$, unit sphere around origin in $\mathbb{R}^{C}$ is denoted as $\mathbb{S}_{C-1}$ and perturb $(\mathbf{p}, \mathbb{D})$ is a function which may push point $\mathbf{p}$ into $\mathbb{D}$ by using boundary reflection. The algorithm of random descent with Lévy flight [9] mutation has three parameters: dimensionless temperature $T>0$, (sub)optimum value $\mathrm{f}^{+} \geq \mathrm{f}^{*}$ and $N$ as the maximum number of $\mathrm{f}(\mathbf{p})$ evaluations.

The algorithm begins from random point $\mathbf{p}_{0} \sim \mathrm{U}(\mathbb{D}), k=0$ and continues for $\mathrm{f}\left(\mathbf{p}_{k}\right)>\mathrm{f}^{+} \wedge k<N$ in loop:

- $d_{k}=\tan \frac{\pi}{2} \eta_{k}, \eta_{k} \sim \mathrm{U}([0,1])$ as Lévy flight length

- $\mathbf{v}_{k} \sim \mathrm{U}\left(\mathbb{S}_{C-1}\right)$ as Lévy flight direction

- $\mathbf{p}_{\text {trial }}=\left[\mathbf{p}_{k}+T d_{k} \mathbf{v}_{k}\right]$ as free point in $\mathbb{Z}^{C}$

- $\mathbf{p}_{\text {new }}=\operatorname{perturb}\left(\mathbf{p}_{\text {trial }}, \mathbb{D}\right)$ as new point in $\mathbb{D}$

- $\mathbf{p}_{k+1}=\left\{\begin{array}{ll}\mathbf{p}_{\text {new }} & \text { for } \mathrm{f}\left(\mathbf{p}_{\text {new }}\right)<\mathrm{f}\left(\mathbf{p}_{k}\right) \\ \mathbf{p}_{k} & \text { for } \mathrm{f}\left(\mathbf{p}_{\text {new }}\right) \geq \mathrm{f}\left(\mathbf{p}_{k}\right)\end{array}\right.$ as stringent decision rule

- $k=k+1$.

This heuristic is relatively simple, but due to Lévy flights [10] it has useful performances.

\section{Referential Measures}

Pearson $\rho_{\mathrm{P}}$, Spearman $\rho_{\mathrm{S}}$ and Kendall $\tau$ correlation coefficients are used as referential SOM quality measures. They are defined as follows

$$
\rho_{\mathrm{P}}=\frac{\sum_{i<j}\left(D_{i j}-\bar{D}\right)\left(L_{i j}-\bar{L}\right)}{\sqrt{\sum_{i<j}\left(D_{i j}-\bar{D}\right)^{2}} \sqrt{\sum_{i<j}\left(L_{i j}-\bar{L}\right)^{2}}},
$$




$$
\begin{gathered}
\rho_{S}=1-\frac{6 \sum_{i<j} r_{i j}^{2}}{n\left(n^{2}-1\right)}, \\
\tau=\frac{n_{c}-n_{d}}{\sqrt{\left(n_{0}-n_{1}\right)\left(n_{0}-n_{2}\right)}},
\end{gathered}
$$

where $\bar{D}=\frac{1}{n} \sum_{i<j} D_{i j} ; \bar{L}=\frac{1}{n} \sum_{i<j} L_{i j} ; n=C(C-1) / 2 ; r_{i j}=x_{i j}-y_{i j}$, i.e. $x_{i j}$ represents rank of sorted Euclidean distances $D_{i j}$, i.e. $y_{i j}$ is rank of sorted graph distances $L_{i j} ; n_{c}$ is the number of concordant pairs; $n_{d}$ is the number of discordant pairs; $n_{0}=n(n-1) / 2 ; n_{1}$ is the number of ties including the vector of all distances $D_{i j}$; and $n_{2}$ is the number of ties including the vector of all distances $L_{i j}$. Finally, quantities $E_{\mathrm{P}}=1-\rho_{\mathrm{P}}, E_{\mathrm{S}}=1-\rho_{\mathrm{S}}$ and $\mathrm{E}_{\mathrm{K}}=1-\tau$ are subjects of discrete minimization.

\section{Case Study: SOM with Hexagonal Topology}

The first aim of our computer experiments was to investigate reliability and time complexity of SOM learning for $\mathbb{A}=\mathbb{B}=\mathrm{V}(\mathcal{G})$. The optimum value of any stress or referential function is $\mathrm{f}^{*}=0$, which is easy to prove. We use hexagonal SOM topology of nineteen vertices $(H=19)$ for seven data points $(C=7)$. Various testing data sets are shown in Fig. 1. Adequate optimization domain $\mathbb{D}$ consist of $19^{7}=893,871,739$ states which are difficult to evaluate systematically. After application of simulated annealing making use of Lévy flights for $T=19, \mathrm{f}^{+}=0, N=30000$, we obtain reliabilities and time complexities for various measures and pattern sets as demonstrated in Tables 1 and 2 . The three most reliable measures are $E_{\mathrm{P}}(19.2 \%), E_{\mathrm{K}}(19.0 \%)$ and $E_{\mathrm{RS}}(17.4 \%)$. Time complexity of SOM learning is small for $E_{\mathrm{RS}}(10526), E_{\mathrm{P}}(10848)$ and $E_{\mathrm{ESA}}(10988)$. Using Feoktistov criterion [11] $F E O=M N E / R E L$, where $M N E$ is the average number of function evaluation for successful trials and $R E L$ is the percentage of successful to total trials, the best measure is $E_{\mathrm{P}}$ with $F E O=56500$ (Table 3).

\begin{tabular}{|c|c|c|c|c|c|c|}
\hline \multirow{2}{*}{ Measure } & \multicolumn{5}{|c|}{ Number of axes } & \multirow{2}{*}{ Average } \\
\hline & 0 & 1 & 2 & $6 \mathrm{~S}$ & $6 \mathrm{~F}$ & \\
\hline$E_{\mathrm{RS}}$ & 12 & 12 & 40 & 14 & 9 & 17.4 \\
\hline$E_{\mathrm{SA}}$ & 11 & 10 & 21 & 13 & 7 & 12.4 \\
\hline$E_{\mathrm{IS}}$ & 9 & 8 & 17 & 13 & 8 & 11.0 \\
\hline$E_{\mathrm{RE}}$ & 9 & 8 & 12 & 14 & 8 & 10.2 \\
\hline$E_{\mathrm{IQ}}$ & 5 & 7 & 8 & 7 & 5 & 6.4 \\
\hline$E_{\mathrm{ESA}}$ & 11 & 12 & 28 & 16 & 8 & 15.0 \\
\hline$E_{\mathrm{P}}$ & 14 & 15 & 39 & 15 & 13 & 19.2 \\
\hline$E_{\mathrm{S}}$ & 14 & 8 & 41 & 10 & 6 & 15.8 \\
\hline$E_{\mathrm{K}}$ & 14 & 9 & 44 & 15 & 13 & 19.0 \\
\hline
\end{tabular}

Table 1: Reliability [\%] of SOM learning for $\mathbb{A}=\mathrm{V}(G)$

\begin{tabular}{|c|c|c|c|c|c|c|}
\hline \multirow{2}{*}{ Measure } & \multicolumn{5}{|c|}{ Number of axes } & \multirow{2}{*}{ Average } \\
\hline & 0 & 1 & 2 & $6 \mathrm{~S}$ & $6 \mathrm{~F}$ & \\
\hline$E_{\mathrm{RS}}$ & 9925 & 10307 & 7497 & 16326 & 8577 & 10526 \\
\hline$E_{\mathrm{SA}}$ & 15286 & 11408 & 12534 & 17749 & 13513 & 14098 \\
\hline$E_{\text {IS }}$ & 10684 & 10771 & 16883 & 13860 & 11141 & 12668 \\
\hline$E_{\mathrm{RE}}$ & 14527 & 16751 & 14323 & 12459 & 14226 & 14457 \\
\hline$E_{\mathrm{IQ}}$ & 12007 & 16813 & 15092 & 15749 & 19160 & 15752 \\
\hline$E_{\mathrm{ESA}}$ & 8545 & 14206 & 8339 & 15182 & 8670 & 10988 \\
\hline$E_{\mathrm{P}}$ & 9997 & 12515 & 8640 & 13925 & 9162 & 10848 \\
\hline$E_{\mathrm{S}}$ & 11049 & 15445 & 9263 & 14026 & 6822 & 11321 \\
\hline$E_{\mathrm{K}}$ & 11207 & 15457 & 9533 & 9745 & 10845 & 11357 \\
\hline
\end{tabular}

Table 2: Time complexity of SOM learning for $\mathbb{A}=\mathrm{V}(G)$ 


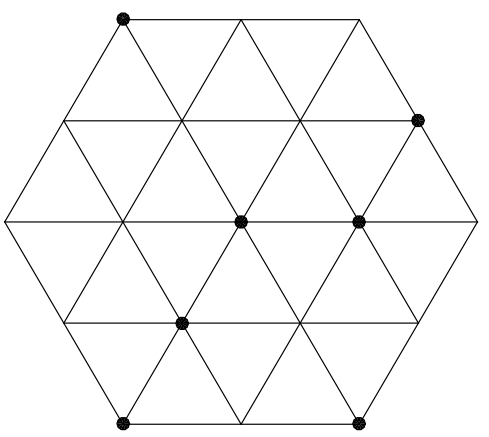

asymmetric case

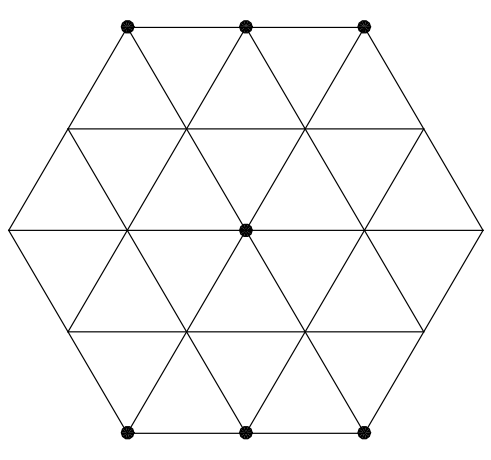

two axes of symmetry
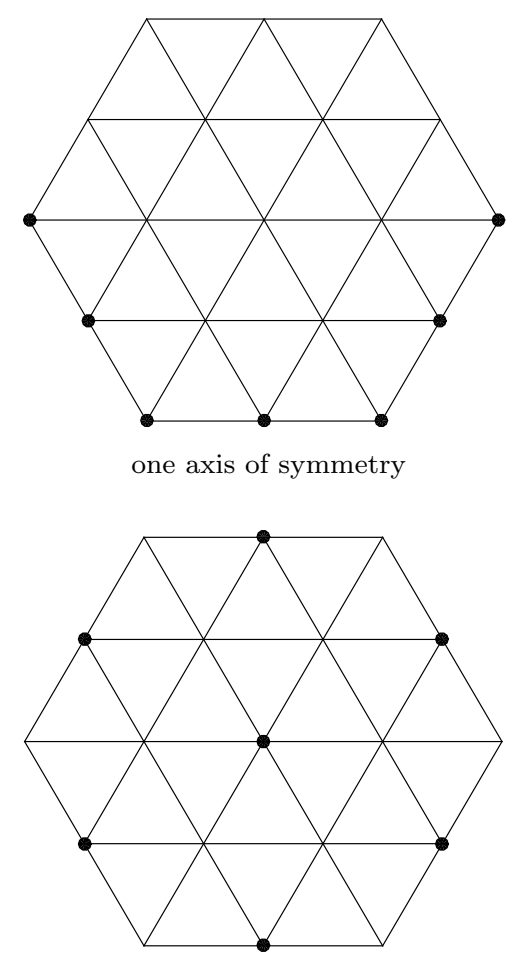

six axes (star)

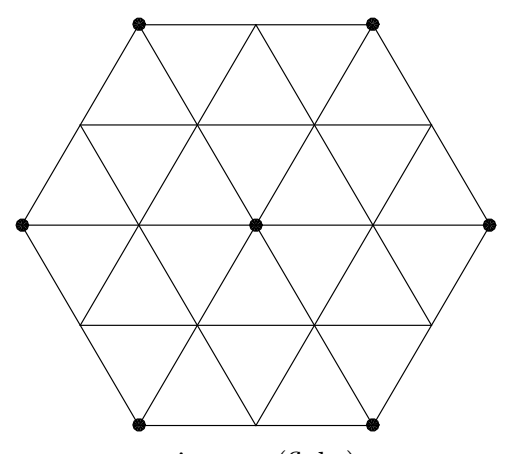

six axes (flake)

Figure 1: Graphical representation of input sets 
Table 3: Feoktistov criterion

\begin{tabular}{c|r|r|}
\multirow{2}{*}{ Measure } & \multicolumn{2}{|c|}{ Mapping from } \\
& \multicolumn{1}{|c|}{$\mathbb{R}^{2}$} & \multicolumn{1}{c|}{$\mathrm{V}(G)$} \\
\hline$E_{\mathrm{RS}}$ & 60494 & 68402 \\
\hline$E_{\mathrm{SA}}$ & 113694 & 89718 \\
\hline$E_{\mathrm{IS}}$ & 115164 & 123698 \\
\hline$E_{\mathrm{RE}}$ & 141735 & 174800 \\
\hline$E_{\mathrm{IQ}}$ & 246125 & 173803 \\
\hline$E_{\mathrm{ESA}}$ & 73253 & 98342 \\
\hline$E_{\mathrm{P}}$ & 56500 & 67791 \\
\hline$E_{\mathrm{S}}$ & 71652 & 97085 \\
\hline$E_{\mathrm{K}}$ & 59774 & 101955 \\
\hline
\end{tabular}

However, a typical application of SOM is mapping from $\mathbb{A}=\mathbb{R}^{M}$ into $\mathbb{B}=\mathrm{V}(\mathcal{G})$ where in our case $M=2$. The optimum stress value is non-negative in general. Data patterns were the same as in the previous case, but their distances were calculated via Euclidean distance from coordinates. The optimum values of measures are included in Table 4. In the second case, reliability and time complexity of SOM learning was studied for $\mathbb{A}=\mathbb{R}^{2}$. After application of simulated annealing making use of Lévy flights for $T=19, H=19, \mathrm{f}^{+}=\mathrm{f}^{*}, N=30000$ and $C=7$, we obtain reliabilities and time complexities for various measures and pattern sets as collected in Tables 5 and 6 . The three most reliable measures are $E_{\mathrm{RS}}(16.4 \%), E_{\mathrm{P}}(15.8 \%)$ and $E_{\mathrm{SA}}(14.2 \%)$. Time complexity of SOM learning is small for $E_{\mathrm{P}}(10711), E_{\mathrm{RS}}(11218)$ and $E_{\mathrm{ESA}}(11801)$. The best measure is again $E_{\mathrm{P}}$ with $F E O=67791$.

Table 4: Optimum values of SOM measures for $\mathbb{A}=\mathbb{R}^{2}$

\begin{tabular}{c|c|c|c|c|c}
\multirow{2}{*}{ Measure } & \multicolumn{6}{|c}{ Number of axes } \\
& 0 & 1 & 2 & $6 \mathrm{~S}$ & $6 \mathrm{~F}$ \\
\hline$E_{\mathrm{RS}}$ & 0.5394 & 0.6026 & 0.7566 & 1.1028 & 0.8616 \\
\hline$E_{\mathrm{SA}}$ & 0.0037 & 0.0039 & 0.0040 & 0.0046 & 0.0049 \\
\hline$E_{\mathrm{IS}}$ & 0.0450 & 0.0445 & 0.0430 & 0.0434 & 0.0452 \\
\hline$E_{\mathrm{RE}}$ & 0.4430 & 0.0389 & 0.0388 & 0.0247 & 0.0285 \\
\hline$E_{\mathrm{IQ}}$ & 0.0662 & 0.0500 & 0.0402 & 0.0209 & 0.0260 \\
\hline$E_{\mathrm{ESA}}$ & 0.1008 & 0.1076 & 0.1204 & 0.1529 & 0.1394 \\
\hline$E_{\mathrm{P}}$ & 0.0124 & 0.0093 & 0.0100 & 0.0279 & 0.0201 \\
\hline$E_{\mathrm{S}}$ & 0.0233 & 0.0146 & 0.0551 & 0.0000 & 0.0339 \\
\hline$E_{\mathrm{K}}$ & 0.0552 & 0.0505 & 0.1205 & 0.0000 & 0.0742 \\
\hline
\end{tabular}

Table 5: Reliability [\%] of SOM learning for $\mathbb{A}=\mathbb{R}^{2}$

\begin{tabular}{|c|c|c|c|c|c|c|}
\hline \multirow{2}{*}{ Measure } & \multicolumn{5}{|c|}{ Number of axes } & \multirow{2}{*}{ Average } \\
\hline & 0 & 1 & 2 & $6 \mathrm{~S}$ & $6 \mathrm{~F}$ & \\
\hline$\overline{E_{\mathrm{RS}}}$ & 11 & 11 & 39 & 11 & 10 & 16.4 \\
\hline$E_{\mathrm{SA}}$ & 10 & 14 & 25 & 12 & 10 & 14.2 \\
\hline$E_{\mathrm{IS}}$ & 11 & 8 & 14 & 9 & 6 & 9.6 \\
\hline$E_{\mathrm{RE}}$ & 10 & 5 & 10 & 10 & 5 & 8.0 \\
\hline$E_{\mathrm{IQ}}$ & 6 & 7 & 13 & 6 & 6 & 7.6 \\
\hline$E_{\mathrm{ESA}}$ & 12 & 9 & 20 & 13 & 6 & 12.0 \\
\hline$E_{\mathrm{P}}$ & 13 & 11 & 26 & 16 & 13 & 15.8 \\
\hline$E_{\mathrm{S}}$ & 11 & 8 & 20 & 11 & 15 & 13.0 \\
\hline$E_{\mathrm{K}}$ & 13 & 8 & 16 & 15 & 15 & 13.4 \\
\hline
\end{tabular}


Table 6: Time complexity of SOM learning for $\mathbb{A}=\mathbb{R}^{2}$

\begin{tabular}{|c|c|c|c|c|c|c|}
\hline \multirow{2}{*}{ Measure } & \multicolumn{5}{|c|}{ Number of axes } & \multirow{2}{*}{ Average } \\
\hline & 0 & 1 & 2 & $6 \mathrm{~S}$ & $6 \mathrm{~F}$ & \\
\hline$E_{\mathrm{RS}}$ & 9357 & 11005 & 5636 & 12093 & 17999 & 11218 \\
\hline$E_{\mathrm{SA}}$ & 5701 & 13073 & 15878 & 15024 & 14024 & 12740 \\
\hline$E_{\text {IS }}$ & 15207 & 10075 & 13316 & 7742 & 13036 & 11875 \\
\hline$E_{\mathrm{RE}}$ & 12591 & 15159 & 15848 & 12569 & 13754 & 13984 \\
\hline$E_{\mathrm{IQ}}$ & 14178 & 16417 & 16208 & 7747 & 11497 & 13209 \\
\hline$E_{\mathrm{ESA}}$ & 14260 & 12554 & 12553 & 14024 & 5615 & 11801 \\
\hline$E_{\mathrm{P}}$ & 12029 & 9837 & 9012 & 9931 & 12747 & 10711 \\
\hline$E_{\mathrm{S}}$ & 12772 & 13749 & 9560 & 13547 & 13479 & 12621 \\
\hline$E_{\mathrm{K}}$ & 16352 & 11780 & 13562 & 9782 & 16836 & 13662 \\
\hline
\end{tabular}

\section{Conclusion}

Six stress measures and three correlation measures were used for batch SOM learning via FSA making use of Lévy flights. Experimental results for mappings from 2D (planar graph and $\mathbb{R}^{2}$ ) to hexagonal grid can be generalized as follows:

In the case of perfect mapping from hexagonal into the same topology recognized $E_{\mathrm{P}}(19.2 \%)$ most reliable for heuristic optimization, while $E_{\mathrm{RS}}(10526)$ measure has less possible time complexity. From the multicriteria decision making theory point of view, both $E_{\mathrm{P}}$ and $E_{\mathrm{RS}}$ are just two Pareto optimal choices. Using Feoktistov criterion, the best value $F E O=56500$ was obtain for Pearson correlation $E_{\mathrm{P}}$. Except for $E_{\mathrm{IQ}}$, the reliability of measures higher than $10 \%$ and their time complexities are comparable.

Similar results were obtained for mapping from $\mathbb{R}^{2}$ into hexagonal topology with just two Pareto optimal choices, namely $E_{\mathrm{RS}}$ with the best reliability $(16.4 \%)$ and $E_{\mathrm{P}}$ with the smallest possible time complexity (10711). Pearson correlation $E_{\mathrm{P}}$ is again the best compromise approach with $F E O=67791$. Except for $E_{\mathrm{IS}}$, $E_{\mathrm{RE}}, E_{\mathrm{IQ}}$, all the measures have higher reliability than $10 \%$ with comparable time complexity.

Therefore, we recommend $E_{\mathrm{RS}}, E_{\mathrm{SA}}, E_{\mathrm{ESA}}$ stress measures and $E_{\mathrm{P}}, E_{\mathrm{S}}, E_{\mathrm{K}}$ correlations for batch SOM learning via FSA making use of Lévy flights.

Acknowledgement: This paper has been supported financially from specific university research MSMT No 21-SVV/2018 and grant GA17-05840S "Multicriteria Optimization of Shift-Variant Imaging System Models" of the Czech Science Foundation.

\section{References}

[1] Kohonen, T.: Self-Organizing Maps, third edn. Springer-Verlag, Berlin, Heidelberg, New York (2001)

[2] Abe, T., Kanaya, S., Kinouchi, M., Kudo, Y., Mori, H., Matsuda, H., Carlos, D.C., and Ikemura, T.: Gene classification method based on batch-learning SOM. Genome Informatics 10, 314-315 (1999)

[3] Kanaya, S., Kinouchi, M., Abe, T., Kudo, Y., Yamada, Y., Nishi, T.,Mori, H., Ikemura, T.: Analysis of codon usage diversity for bacterial genes with a self-organizing map (SOM): characterization of horizontally transferred genes with emphasis on the E. coli O157 genome. Gene 276, 89-99 (2001)

[4] Mojzeš M., Kukal J., Tran, V. Q., Jablonský, J.: Performance comparison of heuristic algorithms via multi-criteria decision analysis. In: R. Matousek (ed.) Proceedings of 17th International Conference on Soft Computing - MENDEL 2011, pp. 244-251. Brno University of Technology, VUT Press, Brno (2011)

[5] Sammon, J.W.: A nonlinear mapping for data structure analysis. IEEE Transaction on Computers C-18(5), 401-409 (1969)

[6] Sun, J., Crowe, M., Fyfe, C.: Extending metric multidimensional scaling with Bregman divergences. Pattern Recognization 44(5), 1137-1154 (2011)

[7] Sun, J., Fyfe, C., Crowe, M.: Extending Sammon mapping with Bregman divergences. Information Sciences 187(1), 72-92 (2012)

[8] Kukal, J.: SOM in Metric Space. Neural Network World 14(6), 469-488 (2004)

[9] Szu, H.: Fast simulated annealing. In: Denker J.S. (ed.) Proceeding of the AIP Conference on Neural Networks for Computing, pp. 420-425. American Institute of Physics, New York (1986)

[10] Viswanathan, G.M., Raposo, E.P., Da Luz, M.G.E.: Lévy flights and superdiffusion in the context of biological encounters and random searches. Physics of Life Reviews 5(3), 133-150 (2008)

[11] Feoktistov, V.: Differential evolution, In Search of Solutions. Springer, Boston, MA (2006) 